\title{
AÇÃo do Trifluralin na Micorrização e Crescimento de Plantas DE AMENDOIM (Arachis hypogaea) ${ }^{1}$
}

\author{
Action of Trifluralin on Mycorrhization and Growth of Peanut (Arachis hypogaea) Plants
}

PEIXOTO, M.F.S.P. ${ }^{2}$, BORGES, V.P. ${ }^{3}$, BORGES, V.P. ${ }^{4}$ e PEIXOTO, C.P. ${ }^{5}$

\begin{abstract}
RESUMO - O amendoim (Arachis hypogaea) é uma planta que durante as fases iniciais do seu desenvolvimento sofre mais intensamente a competição das plantas daninhas, prejudicando sensivelmente sua produção. Uma forma muito eficiente de solucionar esse problema é o uso do herbicida trifluralin; no entanto, seu uso pode prejudicar a micorrização, uma associação benéfica formada por fungos e raízes da planta hospedeira. Este trabalho objetivou avaliar o efeito do herbicida trifluralin e de duas espécies de fungos micorrizicos (Gigaspora margarita e Acaulospora scrubiculata) na micorrização e no crescimento inicial de plantas de amendoim. O trabalho foi realizado na casa de vegetação do Centro de Ciências Agrárias, Ambientais e Biológicas da Universidade Federal do Recôncavo da Bahia, no município de Cruz das Almas - BA. Utilizou-se o cultivar de amendoim Vagem Lisa. Os tratamentos testados envolveram aplicação $(\mathrm{C} / \mathrm{H})$ ou não $(\mathrm{S} / \mathrm{H})$ de herbicida e inoculação individual de dois fungos micorrizicos: Gigaspora margarita (F1) e Acaulospora scrubiculata (F2), sendo os seguintes: S/H $+\mathrm{F} 1, \mathrm{~S} / \mathrm{H}+\mathrm{F} 2, \mathrm{C} / \mathrm{H}+\mathrm{F} 1, \mathrm{C} / \mathrm{H}+\mathrm{F} 2, \mathrm{C} / \mathrm{H} \mathrm{S} / \mathrm{F}$ (sem fungo) e S/H S/F. O delineamento experimental foi inteiramente ao acaso, em esquema fatorial $(2 \times 2)+2$, com quatro repetições. Os resultados mostraram que a colonização micorrizica para Gigaspora margarita foi de $63 \%$ e, quando se aplicou o herbicida, de 44\%; Acaulospora scrubiculata apresentou baixo índice de colonização (5,75\% e $1 \%$, sem e com herbicida, respectivamente); a eficiência micorrizica foi superior na associação com G. margarita; a dependência micorrizica foi aumentada na presença do herbicida para as duas espécies de fungo inoculadas; a altura da planta, o volume de raízes, a massa seca da parte aérea e das raizes e a massa seca da planta foram superiores aos dos demais tratamentos. Conclui-se que o herbicida trifluralin prejudica a micorrização e o crescimento inicial das plantas de amendoim. A espécie de fungo Gigaspora margarita promove o crescimento inicial das plantas de amendoim, porém a espécie Acaulospora scrubiculata não foi eficiente para promover esse crescimento.
\end{abstract}

Palavras-chave: herbicida, micorriza, inoculação.

\begin{abstract}
During their initial developmental stages, peanut (Arachis hypogea) plants suffer weed competition with more intensity, leading to severe losses. A very efficient way to solve this problem is using trifluralin; however, this herbicide can harm mycorrhization, a beneficial association formed by the host plant's fungi and roots. This work aimed to evaluate the effect of trifluralin and of two species of mycorrhizal fungi (Gigaspora margarita and Acaulospora scrubiculata) on mycorrhyzation and the initial growth of peanut plants. The experiment was carried out under greenhouse conditions at the Agricultural, Environmental and Biological Center of the Universidade Federal do Reconcavo Bahiano, in Cruz das Almas-BA, using the peanut cultivar Vagem Lisa. The treatments tested involved application (W/H) ornot (WO/H) of the herbicide and individual inoculation
\end{abstract}

1 Recebido para publicação em 26.11.2009 e na forma revisada em 3.9.2010.

2 Eng ${ }^{\mathrm{a}}-\mathrm{Agr}^{\mathrm{a}}{ }^{-}$, Dr $^{\mathrm{a}}$, Professora Adjunta, Centro de Ciências Agrárias, Ambientais e Biológicas, Universidade Federal do Recôncavo da Bahia - UFRB, 44380-000 Cruz das Almas-BA, <fpeixoto@ufba.br>; ${ }^{3}$ Eng$^{\mathrm{a}}$-Agr ${ }^{\mathrm{a}}$., Doutoranda em Meteorologia, Universidade Federal de Campina Grande, Av. Aprício Veloso, 882, Bairro Universitário, 58424-140 Campina Grande-PB, <valpborges@yahoo.com.br>; ${ }^{4}$ Eng ${ }^{\mathrm{a}}-$ Agr ${ }^{\mathrm{a}}$., Mestranda em Recursos Genéticos Vegetais, Centro de Ciências Agrárias, Ambientais e Biológicas, UFRB, Campus Universitário de Cruz das Almas-BA, <vipborges@yahoo.com.br>; ${ }^{5}$ Engo-Agr ${ }^{0}$., Dr., Professor Associado, Centro de Ciências Agrárias, Ambientais e Biológicas, UFRB, Campus Universitário de Cruz das Almas-BA, <cppeixot@ufrb.edu.br>. 
of two mycorrhizal fungi, Gigaspora margarita $(F 1)$ and Acaulospora scrubiculata (F2), as follows: $W O / H+F 1, W O / H+F 2, W / H+F 1, W / H+F 2, W / H W O / F$ (without the fungus) and WO/ $H W O / F$. The experiment was arranged in a complete randomized block design in a factorial scheme $(2 \times 2)+2$, with four replicates. Results showed that mycorrhizal colonization for Gigaspora margarita was 63\%, and 44\%, when the herbicide was applied; Acaulospora scrubiculata presented low colonization index $15.75 \%$ and $1 \%$, without and with herbicide application, respectively);mycorrhizal efficiency was superiorin the association with G. margarita; mycorrhizal dependence increased in the presence of the herbicide for both the fungi species inoculated; plant height, root volume and root, with upper plant dry mass and plant dry mass being superior in comparison to the other treatments. It was concluded that the herbicide trifluralin harms mycorrhization and the initial growth of peanut plants. The fungus species Gigaspora margarita promoted the initial growth of peanut plants; however, the species Acaulospora scrubiculata was not efficient in promoting this type of growth.

Keywords: herbicide, mycorrhiza, inoculation.

\section{INTRODUÇÃO}

Entre os inúmeros microrganismos que habitam a interface entre as raízes de plantas e o solo, alguns fungos destacam-se, sobremaneira, ao penetrarem nas células vivas da planta hospedeira sem causar danos. A associação simbiótica formada pelo fungo com as raízes da planta hospedeira, conhecida como micorriza (do grego: $m y k e=$ fungo e $r h i z a=$ raiz), caracteriza-se pela condição mutualística, uma vez que ambos os organismos se beneficiam da associação e, portanto, devem ser estudados como um sistema dinâmico e não como organismos individualizados (Oliveira \& Trindade, 2000). Essa associação aumenta a capacidade das plantas em absorver nutrientes,melhorando sua resposta aos fertilizantes e corretivos e beneficiando seu crescimento e produção (Miranda \& Miranda, 2002).

Diversos fatores afetam o processo de micorrização, entre eles a utilização de pesticidas, mais especificamente herbicidas. Esses efeitos variam com o tipo de herbicida (propriedades físico-químicas das moléculas), tipo de solo, sistema de cultivo, espécie de fungo e da planta estudada (Bethlenflavay et al., 1996; Santos et al., 2006; Dawo et al., 2009). Vários trabalhos avaliam essa questão com enfoques diferentes, e os resultados são variáveis. Silva et al. (2006) verificaram, em experimento em casa de vegetação com a cultura da soja, que na última época de dessecação ou roçada de Brachiaria brizantha (sete dias) o herbicida glifosato promoveu inibição na micorrização quando se inocularam as espécies Glomus etunicatum, Glomus fasciculatum e Scutelospora heterogama. Em contrapartida, Malty et al. (2006) não observaram esse efeito inibitório do glyphosate (doses de 1,25 a $10 \mathrm{~L} \mathrm{ha}^{-1}$ ) na colonização de plantas de soja pelos fungos Gigaspora margarita, Glomus etunicatum e Scutelospora heterogama. Vieira et al. (2007) avaliaram o efeito do herbicida sulfentrazone em processos simbióticos associados à planta de soja em condições de casa de vegetação e observaram que houve redução de $50 \%$ na porcentagem de infecção dos fungos micorrízicos e diminuição no crescimento inicial da planta. Abd-Alla et al. (2000), estudando o efeito de gramoxone e brominal sobre a taxa de colonização e o número de esporos de fungos micorrizicos em Vigna sinensis, Phaseolus vulgaris e Lupinus albus, verificaram que essas moléculas inibiram a taxa de colonização e afetaram o crescimento da planta; o efeito foi variável, com o pesticida e a espécie estudada. Da mesma forma, efeitos variáveis, em função da aplicação de herbicidas, sobre a colonização micorrízica também foram encontrados por Reis et al. (2009) quando avaliaram o efeito do ametryn, tryfloxysulfuronsodium, ametryn + tryfloxysulfuron-sodium e sulfentrazone em dois cultivares de cana-deaçúcar, em campo.

Siqueira et al. (1991) observaram que a aplicação de imazethapyr e pendimethalin, nas maiores doses indicadas, reduziu a taxa de colonização de fungos micorrízicos arbusculares em plantas de sorgo. No entanto, Su-Chen et al. (2000), testando o efeito de três diferentes herbicidas com os ingredientes ativos: 
(isopropilamina $\mathrm{N}$ - fosfometil glicina), (1,1dimetil -4,4 -bipiridium dicloro) e (bensulfuron metil benzoato) na colonização e produção de esporos de fungos endomicorrízicos arbusculares, concluíram que todos os herbicidas testados não exerceram impacto imediato sobre a comunidade desses fungos. Em contrapartida, Kumar et al. (1999) verificaram que o 2,4-D aumentou a porcentagem de infecção e o número de propágulos de fungos micorrízicos arbusculares em duas espécies de plantas de um ecossistema agroflorestal.

O amendoim é uma planta dicotiledônea, da família Leguminosae, que sofre a concorrência competitiva das plantas daninhas, principalmente nas fases iniciais do seu desenvolvimento. O controle químico é realizado com herbicidas, entre eles a trifluralina - do grupo químico das dinitroanilinas (Sader et al., 1979).

$\mathrm{Na}$ Bahia, mais especificamente na região do Recôncavo, não há relatos de estudos relacionados com esses aspectos na cultura do amendoim. Dessa forma, torna-se de grande importância a realização de pesquisas quanto aos aspectos considerados, levando-se em conta que são inexistentes nessa região. Assim, objetivou-se avaliar o efeito do herbicida trifluralin e de duas espécies de fungos micorrízicos (Gigaspora margarita e Acaulospora scrubiculata) na micorrização e no crescimento inicial de plantas de amendoim.

\section{MATERIAL E MÉTODOS}

O experimento foi realizado na casa de vegetação do Centro de Ciências Agrárias, Ambientais e Biológicas da Universidade Federal do Recôncavo da Bahia, no município de Cruz das Almas-BA, no período de junho a dezembro de 2008. O plantio foi efetuado em vasos com capacidade para $5 \mathrm{~kg}$ com o cultivar de amendoim Vagem Lisa. Utilizou-se como substrato material de solo retirado da camada de 0,0-0,2 m de um Latossolo Amarelo do Recôncavo Baiano.

Os tratamentos testados envolveram aplicação $(\mathrm{C} / \mathrm{H})$ ou não $(\mathrm{S} / \mathrm{H})$ de herbicida e inoculação individual de dois fungos micorrízicos: Gigaspora margarita (F1) e Acaulospora scrubiculata (F2), sendo os seguintes: $\mathrm{S} / \mathrm{H}+$ $\mathrm{F} 1, \mathrm{~S} / \mathrm{H}+\mathrm{F} 2, \mathrm{C} / \mathrm{H}+\mathrm{F} 1, \mathrm{C} / \mathrm{H}+\mathrm{F} 2, \mathrm{C} / \mathrm{H} \mathrm{S} / \mathrm{F}$ (sem fungo) e $\mathrm{S} / \mathrm{H} \mathrm{S} / \mathrm{F}$. O delineamento experimental foi inteiramente ao acaso, em esquema fatorial $(2 \times 2)+2$, com quatro repetições.

Antes da instalação do ensaio, o solo foi seco ao ar, peneirado em peneira de malha de $2 \mathrm{~mm}$ de diâmetro e autoclavado a $120^{\circ} \mathrm{C}$, $1 \mathrm{~atm}$, durante uma hora e $20 \mathrm{~min}$, por duas vezes (Menezes \& Hanlin, 1997). Utilizou-se o herbicida trifluralina (grupo quimico das dinitroanilinas) na dose de $1,5 \mathrm{~L} \mathrm{ha}^{-1}$, de acordo com a recomendação de Rodrigues \& Almeida (1998) para a cultura e o tipo de solo em questão, aplicado em pré-plantio incorporado, misturando-se o herbicida com uma camada de solo de aproximadamente 7 a $10 \mathrm{~cm} \mathrm{-} \mathrm{a}$ mesma que atinge a grade quando da incorporação deste herbicida no campo. Os inóculos das duas espécies de fungo micorrízicos foram obtidos da coleção do Laboratório de Microbiologia do Solo da Embrapa Mandioca e Fruticultura. A produção é feita através da multiplicação dos esporos das referidas espécies, em substrato estéril, na presença de Brachiaria decumbens, contendo no mínimo 148 esporos em $30 \mathrm{~g}$ de inóculo. A inoculação foi realizada com o solo rizosférico contendo raízes colonizadas de Brachiaria e esporos dos fungos micorrizicos, no dia do plantio, antes da colocação das sementes e na proporção de 20 g por cova.

Ao final de 45 dias, as plantas foram cuidadosamente retiradas dos vasos e levadas ao laboratório, para avaliação das seguintes características: massa seca da parte aérea e da raiz (obtida com estufa a $60^{\circ} \mathrm{C}$ ), altura total da planta $(\mathrm{cm})$ e volume de raízes. O volume de raízes foi determinado com auxílio de uma proveta de $100 \mathrm{~mL}$, a qual foi completada com água; posteriormente, fez-se a imersão da raiz, previamente lavada e seca. Com uma pipeta graduada de $5 \mathrm{~mL}$, foi retirado e anotado o excesso de água que ultrapassou a capacidade da proveta, o qual correspondia ao volume da raiz, considerando-se que $1 \mathrm{~mL}=1 \mathrm{~cm}^{3}$. Os cálculos da dependência micorrízica e da eficiência micorrízica foram efetuados de acordo com o método proposto por Planchette et al. (1983) e Soares et al. (2003), respectivamente. A colonização micorrízica foi avaliada através das raízes separadas do solo e submetidas à despigmentação em $\mathrm{KOH}(10 \%)$ e posterior 
coloração em fucsina ácida 0,05\% (Phillips \& Hayman, 1970), determinando-se o percentual de colonização radicular pelo método da contagem em placa reticulada (Ambler \& Young, 1977). A análise da variância foi feita de acordo com o modelo do experimento (inteiramente casualizado em esquema fatorial), utilizandose o programa SAS System for Windows; no entanto, o teste de Tukey a 5\% de probabilidade só foi efetuado quando a interação foi significativa.

\section{RESULTADOS E DISCUSSÃO}

Na Figura 1A, B e C encontram-se os resultados da colonização, eficiência e dependência micorrizica, respectivamente. A colonização micorrízica para Gigaspora margarita foi de $63 \%$ e, quando se aplicou o herbicida, $44 \%$ (Figura 1A). Acaulospora scrubiculata apresentou baixo índice de colonização $(5,75 \%$ e $1 \%$, sem e com herbicida, respectivamente). A interação entre plantas e fungos micorrízicos tem início antes mesmo do estabelecimento de um contato físico, por meio de trocas de sinais moleculares. Os exsudatos das raizes das plantas capazes de se associar com fungos micorrizicos contêm substâncias que podem estimular a germinação de esporos e o crescimento do fungo, ao contrário daquelas não hospedeiras, que não possuem essas substâncias, e sim compostos antifúngicos (Moreira \& Siqueira, 2002). Possivelmente, os exsudatos das raízes do amendoim não estimularam a colonização de $A$. scrubiculata. Quanto à ação do herbicida, que reduziu a colonização de ambas as espécies de fungos micorrízicos, Rodrigues \& Almeida (1995) explicam que esse fato pode estar relacionado ao comprometimento do processo de divisão celular da planta, em função do mecanismo de ação do trifluralin, que prejudica a divisão celular das células das raízes. Provavelmente, não houve desenvolvimento suficiente do sistema radicular para o estabelecimento dos fungos, que necessitam da presença de raízes vivas para se multiplicar e, com isso, beneficiar a planta com essa associação mutualística, ou o próprio herbicida teve ação maléfica sobre as espécies de fungo estudadas.

Essencialmente, a eficiência micorrízica é a habilidade do fungo de aumentar a fotossíntese e o crescimento do hospedeiro, através de um melhor fornecimento de nutrientes, principalmente $\mathrm{P}$ (Matos et al., 1999). Essa variável - que é determinada com os valores de matéria seca da parte aérea - mostrou-se também superior na associação com G. margarita. Como a eficiência do inóculo depende da compatibilidade do fungo inoculante com a planta hospedeira (Zambolim \& Siqueira, 1985), a associação com $A$. scrubiculata não foi eficiente em promover o crescimento das plantas estudadas (Figura 1B). Houve aumento da dependência micorrízica na presença do herbicida para as duas espécies de fungo inoculadas. Esse resultado pode ser explicado em função do mecanismo de ação do herbicida, que, afetando a divisão celular do meristema radicular (Vidal \& Fleck, 2001), diminui o volume das raizes e, consequentemente, sua área de exploração. Dessa forma, a planta torna-se mais dependente da associação micorrízica (Figura 1C).
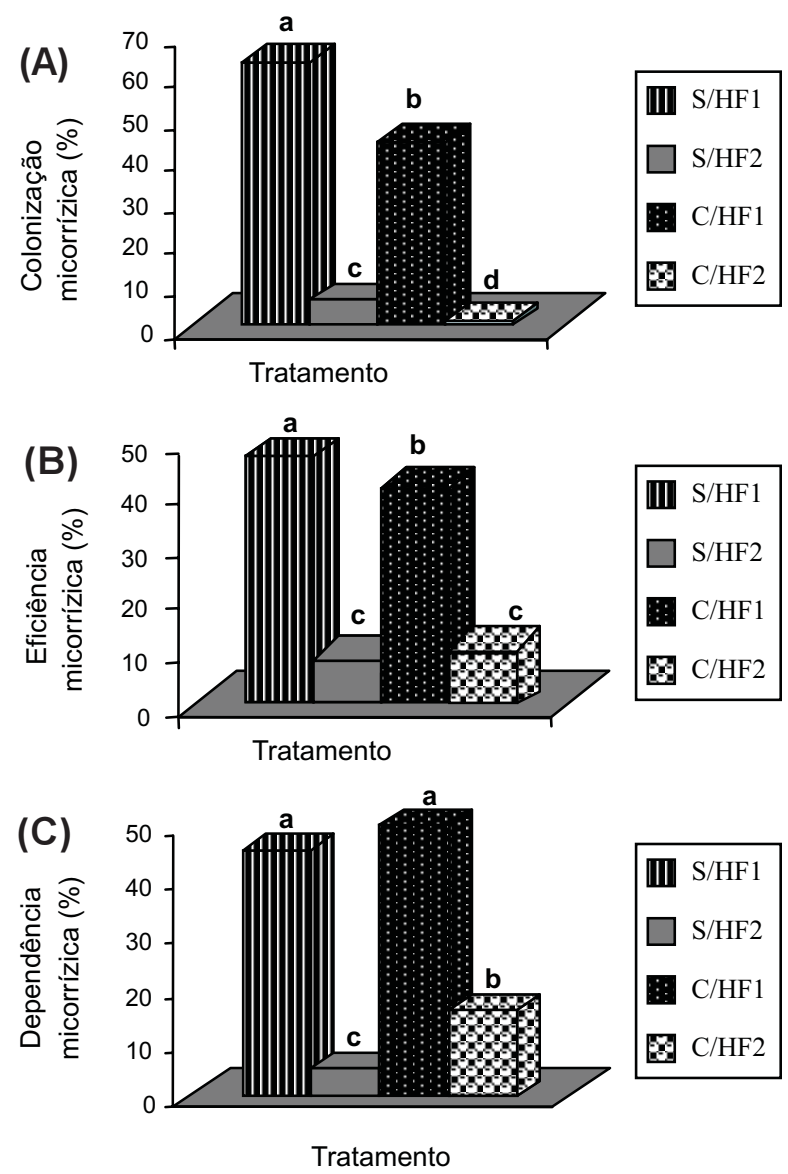

Figura 1 - Colonização (A), eficiência (B) e dependência micorrízica (C), em plantas deamendoim inoculadas com Gigaspora margarita (F1) e Acaulospora scrubiculata (F2), na presença $(\mathrm{C} / \mathrm{H})$ e ausência $\mathrm{S} / \mathrm{H})$ de herbicida trifluralin. 
Observa-se, na Figura 2, que as variáveis altura da planta, volume de raízes e matéria seca da planta (total, parte aérea e raízes) apresentaram comportamento semelhante diante dos tratamentos testados. As plantas inoculadas com Gigaspora margarita obtiveram melhores resultados mesmo no tratamento com herbicida. Isso demonstra o efeito benéfico do fungo à planta, o qual, pelas suas hifas externas, aumentou o volume de solo explorado pelas plantas, potencializando a absorção de nutrientes de baixa mobilidade no solo, como P, Zn e Cu (Marschner \& Dell, 1994). A inoculação com Acaulospora scrubiculata não foi efetiva para o desenvolvimento da planta, já que se verificaram resultados semelhantes aos obtidos no tratamento testemunha $(\mathrm{S} / \mathrm{H}$ $\mathrm{S} / \mathrm{F})$.

Independentemente da espécie micorrízica inoculada, a aplicação do herbicida provocou redução nas variáveis supracitadas. Esse herbicida une-se fortemente à tubulina, proteína que faz parte dos microtúbulos, responsáveis pela movimentação dos cromossomos durante a divisão celular. Consequentemente, não há movimentação dos cromossomos e a divisão celular é interrompida na prófase. Os meristemas das raízes ficam atrofiados e as plântulas apresentam reduzida capacidade de absorção de água e nutrientes, morrendo antes de emergirem do solo. As que conseguem emergir podem apresentar caule "inchado" na região de contato com o solo (Vidal \& Flack, 2001). Efeitos na redução no crescimento inicial de plantas de soja foram observados por Vieira et al. (2007), quando utilizaram o herbicida sulfentrazone. Silva et al. (2006) verificaram efeito inibitório do glyphosate, quando utilizado na última época de dessecação de Brachiaria brizanta, na micorrização de plantas de soja inoculadas com os fungos Glomus etunicatum, Glomus fasciculatum e Scutelospora heterogama,

Conclui-se que o herbicida trifluralin prejudica a micorrização e o crescimento inicial
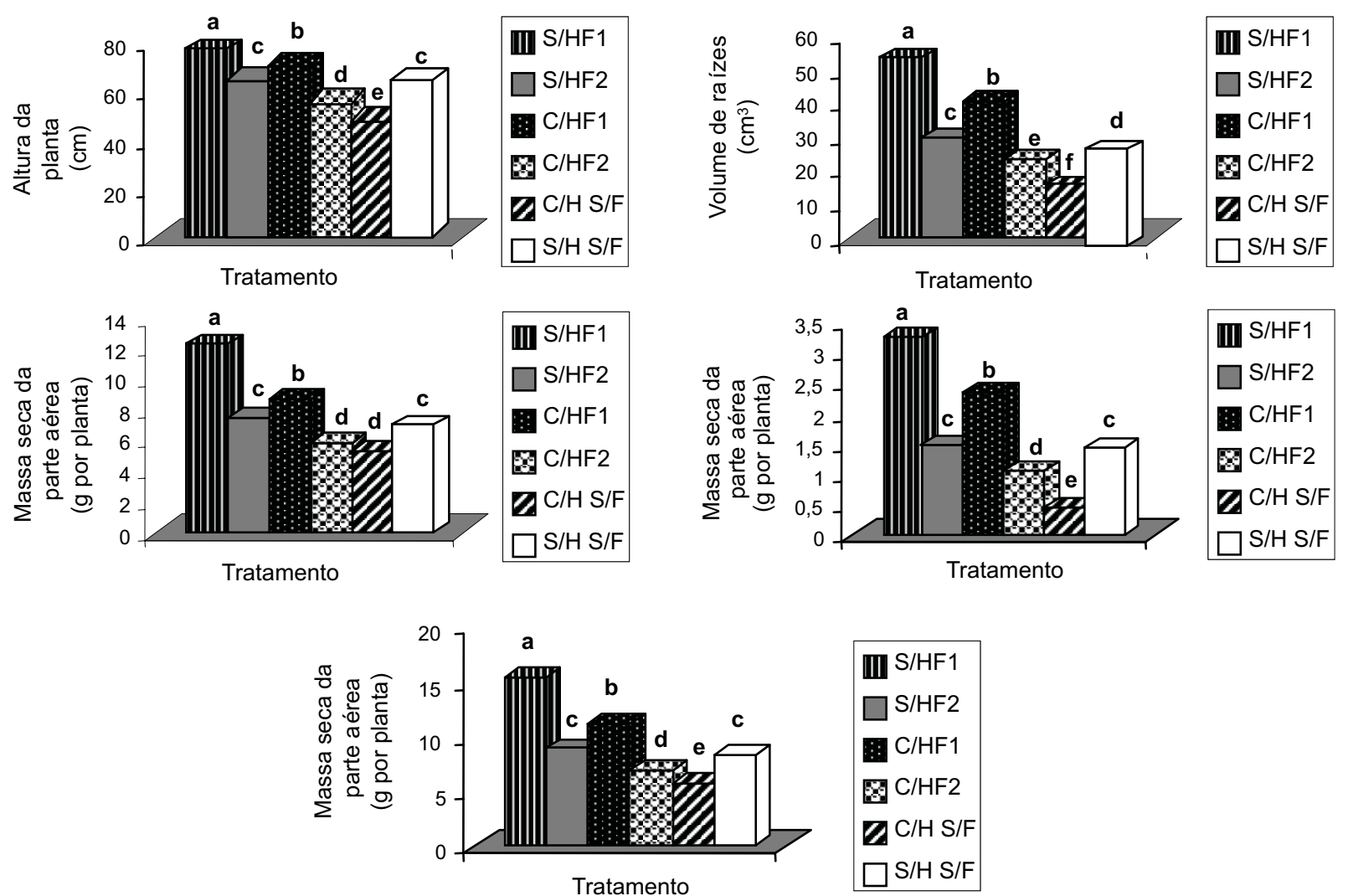

Figura 2 - Altura da planta, volume de raízes e matéria seca (parte aérea, raízes e total) em plantas de amendoim inoculadas com Gigaspora margarita (F1) e Acaulospora scrubiculata (F2), ou não inoculadas (S/F), na presença (C/H) e ausência S/H) de herbicida trifluralin. 
das plantas de amendoim. O fungo Gigaspora margarita promove o crescimento inicial das plantas de amendoim; no entanto, o fungo Acaulospora scrubiculata não foi efetivo em promover esse crescimento.

\section{LITERATURA CITADA}

ABD-ALLA, M. H.; OMAR, S. A.; KARANXHA, S. The impact of pesticides on arbuscular mycorrhizal and nitrogenfixing symbioses in legumes. Appl. Soil Ecol., v. 14, n. 3, p. 191-200, 2000.

AMBLER, J. R.; YOUNG, J. L. Technics for determining root lenght infected by vesicular-arbuscular mycorrizae. Soil Sci. Soc. Am. J., v. 4, p. 551-556, 1977.

BETHLENFALVAY, G. J. et al. Mycorrhizae, biocides, and biocontrol. Herbicide-mycorrhiza interactions in soybean and cocklebur treated with bentazon. Appl. Soil Ecol., v. 3, n. 2, p. 197-204, 1996.

DAWO, M. I.; WILKINSON, J. M.; PILBEAM, D. J. Interactions between plants in intercropped maize and common bean. J. Sci. Food Agric., v. 89, n. 1, p. 41-48, 2009

KUMAR, P. P.; REDDY, S. R.; REDDY, S. M. Interaction of some agrochemicals and VAM-fungi on growth of two agroforest tree species in nursery. J. Mycol. Plant Pathol., v. 29, n. 3 , p. $385-388,1999$

MALTY, J. S.; SIQUEIRA, J. O.; MOREIRA, F. M. S. Efeitos do glifosato sobre microrganismos simbiotróficos de soja, em meio de cultura e casa de vegetação. Pesq. Agropec. Bras., v. 41, n. 2, p. 285-291, 2006.

MENEZES, M.; HANLIN, D. N. W. S. Guia prático para fungos fitopatogênicos. Recife: Imprensa Universitária UFRP, 1997. $106 \mathrm{p}$.

MIRANDA, J. C. C.; MIRANDA, L. N. Importância da micorriza arbuscular para o cultivo da soja na região do cerrado. Planaltina: Ministério da Agricultura, Pecuária e Abastecimento, 2002. 5 p. (Comunicado Técnico, 75)

MATOS, R. M. B.; SILVA, E. M. R.; LIMA, E. Fungos micorrízicos e nutrição de plantas. Seropédica: Embrapa Agrobiologia, 199936 p. (Embrapa-CNPAB. Documentos, 98)

MARSCHNER, H.; DELL, B. Nutrient uptake in mycorrhizal symbiosis. Plant Soil, v. 159, n. 1, p. 89-102, 1994

MOREIRA, F. M. S.; SIQUEIRA, J. O. Microbiologia e bioquímica do solo. Lavras: Universidade Federal de Lavras, 2002. $626 \mathrm{p}$.

OLIVEIRA, A. A. R.; TRINDADE, A. V. Micorrizas na agricultura. 2000. Disponível em: $<\mathrm{http}$ //

www23. sede.embrapa.br:8080/aplic/rumos.nsf/ blbbbc852ee1057183256800005ca0ab/ e3c77421113091a903256c2b0047ed54?OpenDocument>. Acesso em: 9 jan. 2010.

Planta Daninha, Viçosa-MG, v. 28, n. 3, p. 609-614, 2010
PHILLIPS, J. M.; HAYMAN, D. S. Improved procedures for clearning roots and staining parasitic and vesicular-arbuscular mycorrhyzal fungi for rapid assessement of infection. Trans. Br. Mycol. Soc., v. 55, p. 158-161, 1970.

PLANCHETTE, C.; FORTIN, J.A.; FURLAN, V. Growth response of several plants species to mycorrhiza in a soil of moderate fertility.I. Mycorrhizal dependence under field conditions. Plant Soil, v. 70, p. 191-209, 1983

REIS, M. R. et al. Colonização micorrízica e atividade de fosfatases ácidas na rizosfera de cultivares de cana-de-açúcar após a aplicação de herbicidas. Planta Daninha, v. 27, p. 977-985, 2009. (Número Especial)

RODRIGUES, B. N.; ALMEIDA, F. S. Guia de herbicidas. 4.ed. Londrina: Edição dos Autores, 1998. 648 p.

SADER, R.; CARVALHO, N. M.; CAMARGO, M. Efeito de diferentes doses de trifluralina no poder germinativo e produção de sementes de amendoim. R. Bras. Sementes, v. 1, p. $59-63,1979$

SANTOS, J. B. et al. Action of two herbicides on the microbial activity of soil cultivated with common bean (Phaseolus vulgaris) in conventional-till and no-till systems Europ. Weed Res. Soc., v. 46, n. 4, p. 284-289, 2006.

SILVA, A. C. et al. Micorrização e épocas de dessecação de Brachiaria brizanta no desenvolvimento da soja. Planta Daninha, v. 24, n. 2, p. 271-277, 2006

SILVA, J. B. C.; NAKAGAWA, J. Estudo de fórmulas para cálculo da velocidade de germinação. Inf. Abrates, v. 5, n. 1, p. $62-73,1995$

SIQUEIRA, J. O.; SAFIR, G. R.; NAIR, M. G. Vamycorrhizae and mycorrhiza stimulating isoflavnoid compounds reduce plant herbicide injury. Plant Soil, v. 134, n. 2, p. 233-242, 1991.

SOARES, A. C. F. et al. Produção de mudas de ipê roxo inoculadas com fungos micorrízicos arbusculares. Magistra, v. 15, n. $2,123-127,2003$.

SU-CHEN, L.; SU-YANG, L.; CHI-GUANG, W. Herbicide a stress factor on the population flutuaction of arbuscular mycorrhizae fungi. J. Agric. Res. China, v. 49, n. 1, p. $42-49,2000$

VIEIRA, R. F.; SILVA, C. M. M. S.; SLVEIRA, A. P. D. Soil microbial biomass $\mathrm{C}$ and symbiotic processes associated with soybean after sulfentrazone herbicide application. Plant Soil, v. 300, n. 1-2, p. 91-103, 2007.

VIDAL, R. A.; FLECK, N. G. Inibidores da polimerização da tubulina. In: VIDAL, R. A.; MEROTO Jr., A. (Eds.) Herbicidologia. Porto Alegre: Evangraf, 2001. p. 131-137.

ZAMBOLIM, L.; SIQUEIRA, J. O. Importância e potencial das associações micorrízicas para a agricultura. Belo Horizonte: EPAMIG, 1985. 36 p. (Série Documentos, 26) 